\title{
Das Change Management weiterentwickeln
}

\section{Aktuelle Herausforderungen, Erfolgsfaktoren und Anpassungen für die digitale Transformation}

\author{
Florian Dörries, Marco Wichering und Wolfgang Kersten, Technische Universität Hamburg
}

Evolving Change Management: Current Challenges, Success Factors and Adjustments for Digital Transformation

In view of the digital transformation, companies are facing radical changes which could have a big impact on their business success. Change management could help companies to deal with this transformation and to avoid the risk of losing market share to more innovative companies. The more important question at this stage is if traditional Change management models handle this topic well or if they must be adjusted. This article identifies challenges and success factors by using a literature analysis and a survey, by means of which an adapted model for successful Change management is generated.

Keywords:

transformation, digitalization, implementation

\begin{abstract}
Unternehmen stehen vor dem Hintergrund der digitalen Transformation vor einschneidenden Veränderungen, die den Unternehmenserfolg beeinflussen können. Change Management kann die Unternehmen unterstützen, diese Transformation erfolgreich umzusetzen und somit der Gefahr zu entgehen, bspw. Marktanteile an innovationskräftigere Unternehmen zu verlieren. Die damit einhergehende Fragestellung ist allerdings, inwiefern die tradierten Modelle des Change Managements passend für den anstehenden Wandel sind und ob diese weiterentwickelt werden müssen. In diesem Beitrag werden mithilfe einer Literaturanalyse sowie einer Befragung aktuelle Herausforderungen und Erfolgsfaktoren des Change Managements identifiziert, wodurch im Anschluss ein angepasstes Modell für erfolgreiche Change-Projekte abgeleitet werden kann.
\end{abstract}

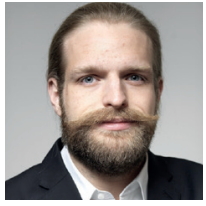

Florian Dörries, M. Sc. arbeitet als wissenschaftlicher Mitarbeiter an der Technischen Universität Hamburg am Institut für Logistik und Unternehmensführung.

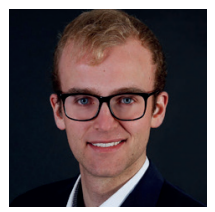

Marco Wichering, M. Sc. arbeitet derzeit in einer Unternehmensberatung und war Masterand an der Technischen Universität Hamburg am Institut für Logistik und Unternehmensführung.

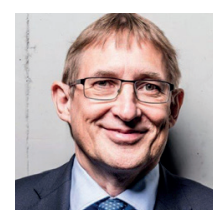

Prof. Dr. Dr. h. c. Wolfgang Kersten leitet an der Technischen Universität Hamburg das Institut für Logistik und Unternehmensführung.

logu@tuhh.de

www.tuhh.de/logu
Digitalisierung, digitale Transformation und Industrie 4.0 sind Themenfelder, die seit Jahren in der Unternehmenswelt diskutiert werden. Abgesehen von eher wenigen (Leuchtturm-) Projekten einzelner Unternehmen scheitert in der deutschen Arbeitswelt die Digitalisierung häufig an guten Vorsätzen, deren Realisierung oftmals aufgeschoben wird [1]. So konnte bspw. die Studie "Trends und Strategien in Logistik und Supply Chain Management" der Bundesvereinigung Logistik identifizieren, dass die Digitalisierung nach wie vor das beherrschende Thema ist, der Fortschritt bei der digitalen Transformation im Vergleich zu 2016 weiterhin begrenzt bleibt [2].

Im März 2020 traf die Corona-Krise Deutschland und führte notgedrungen zu dem langersehnten Schub bei der digitalen Transformation der Unternehmen [3]. Es ist mehr Mitarbeitern plötzlich möglich Vollzeit im Home-Office zu arbeiten, zudem entwickeln mehr Unternehmen eigene digitale Vertriebswege, der Datenaustausch - um das Supply Chain Risk Management zu verbessern - wird gesteigert etc. [4]. Viele Digitalisierungsmaßnahmen in der Corona-Krise sind sicherlich kurzfristig bzw. aus der
Not heraus entstanden, dennoch wird deutlich, dass in der Vergangenheit nicht die Technik der alleinige entscheidende restriktive Faktor war, sondern vielmehr der Willen zur Umsetzung bzw. zum Wandel.

Mittlerweile sind die Unternehmen weitestgehend in der Lage, ihre Digitalisierungs-Aktivitäten aus der Corona-Krise zu reflektieren und mit Blick auf eine langfristige Strategie anzupassen. Dabei stellt sich nach wie vor jedoch die Frage, was die richtigen Schritte sind, die bei der Umsetzung berücksichtigt werden müssen, um einen langfristigen Erfolg zu versprechen. Change Management als etabliertes Konzept bietet hierfür Ansatzpunkte. Dabei wird es in diesem Zusammenhang als Funktion verstanden, die "dem ganzheitlichen und systematischen Planen, Initiieren, Realisieren, Reflektieren und Stabilisieren von Veränderungsprozessen in Organisationen" dient [5]. Im vorliegenden Beitrag wird dieses Change Management im Kontext der digitalen Transformation untersucht. Die digitale Transformation umfasst aus der Unternehmensperspektive den Wandel von Wertschöpfungsprozessen durch die Einführung digitaler Technologien 


\section{Teilnehmer nach Unternehmensgröße}

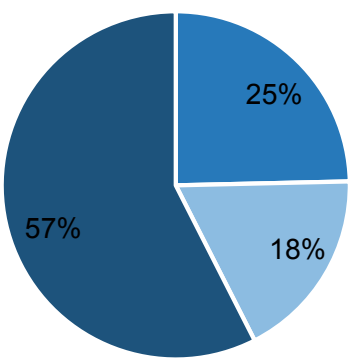

- kleine Unternehmen (bis 49 MA)

mittlere Unternehmen (bis 249 MA)

Bild 1: Sample der empirischen Studie $(n=207)$.

(Implementierung neuer bzw. Weiterentwicklung bestehender), die Anpassung der Unternehmensstrategie (basierend auf neuen digitalisierten Geschäftsmodellen) und den Aufbau entsprechender Kompetenzen [6]. Im Folgenden wird das Change Management hinsichtlich der Angemessenheit und möglicher notwendiger Anpassungen untersucht.

\section{Forschungsansatz}

Mit dem Ziel neben dem Umsetzungsstand sowie Handlungsbedarfen ebenfalls die aktuellen Herausforderungen und Erfolgsfaktoren im Kontext der digitalen Transformationen zu identifizieren, wurde eine empirische Studie in Form einer Befragung durchgeführt. Bei dieser waren die Antwortmöglichkeiten mithilfe von Multiple-Choice-Auswahlmöglichkeiten bzw. Likert-Skalen vorgegeben. Die Studie fußt auf den Erkenntnissen einer strukturierten Literaturanalyse, in welcher $u$. a. die drei Kernelemente erfolgreicher Veränderungsprozesse - Strategie, Kommunikation und Mitarbeitereinbindung - identifiziert werden konnten. Die Antwortmöglichkeiten zu den in der Befragung thematisierten und hier in erster Linie diskutierten Erfolgsfaktoren und Herausforderungen basieren $u$. a. auf den Erkenntnissen von Bowersox u. a., Kane u. a. und Lauer [7-9]. Eine tiefergehende Darstellung der Literaturanalyse wird an dieser Stelle ausgespart.

Die Datenerhebung fand in zwei Phasen - Januar und Februar sowie Juli bis September 2020 - in Form einer Online-Befragung statt. Die Zielgruppe umfasste Unternehmensvertreter, die in der Vergangenheit bereits in einen Veränderungsprozess involviert waren. Die Teilnehmer-Akquise erfolgte in thematisch passenden Gruppen der sozialen Netzwerke
XING und LinkedIn sowie per Direktansprache. Nach Abschluss der Datenerhebung und Datenbereinigung lagen insgesamt 207 verwertbare Antwortbögen vor, welche u. a. die Grundlage für die folgenden Erkenntnisse bilden. Im Rahmen dieses Beitrags wird nur ein Auszug der Befragungsergebnisse dargestellt. Die Zusammensetzung des Samples ist Bild 1 zu entnehmen.

Das klassische Change Management ist nicht genug

Grundsätzliche Herausforderungen in der praktischen Umsetzung des Change Managements lassen sich an der verhältnismäßig geringen Erfolgsquote bemessen. So gaben lediglich $31 \%$ der Befragten an, dass die Change-Projekte in ihren Unternehmen im Kontext der digitalen Transformation in hohem bzw. sehr hohem Maße erfolgreich waren. Diese Erkenntnis deckt sich mit weiteren Studien, die unabhängig von der digitalen Transformation zu dem Ergebnis kommen, dass ca. 2/3 aller Change-Projekte scheitern [10-12].

Abgesehen von der anhaltenden scheinbar geringen Erfolgsquote lässt sich die Kritik an den tradierten Modellen des Change Managements üben, dass diese den Anforderungen des Wandels im Kontext der digitalen Transformation nicht vollständig gerecht werden [13]. So gehen $70 \%$ der Befragten davon aus, dass das Change Management angepasst werden muss, um die digitale Transformation besser unterstützen zu können. Um diesen Umstand zu verdeutlichen wird im Folgenden eine aggregierte Sicht der Phasen bzw. Schritte der tradierten Change-Modelle von Lewin, Kotter und Krüger [14-16] vorgestellt. Wie in Bild 2 dargestellt, lassen sich die Modelle in die folgenden fünf Phasen aufteilen: Bedarf identifizieren, Führungsteam aufbauen, Strategie entwickeln \& kommunizieren, Veränderungen umsetzen und Veränderungen leben.

Um festzustellen, inwieweit Anpassungen am Change Management vorzunehmen sind, damit der digitalen Transformation entsprochen werden kann, ist ein Blick auf ebendiese unumgänglich. Beispielsweise benennen Schu- 


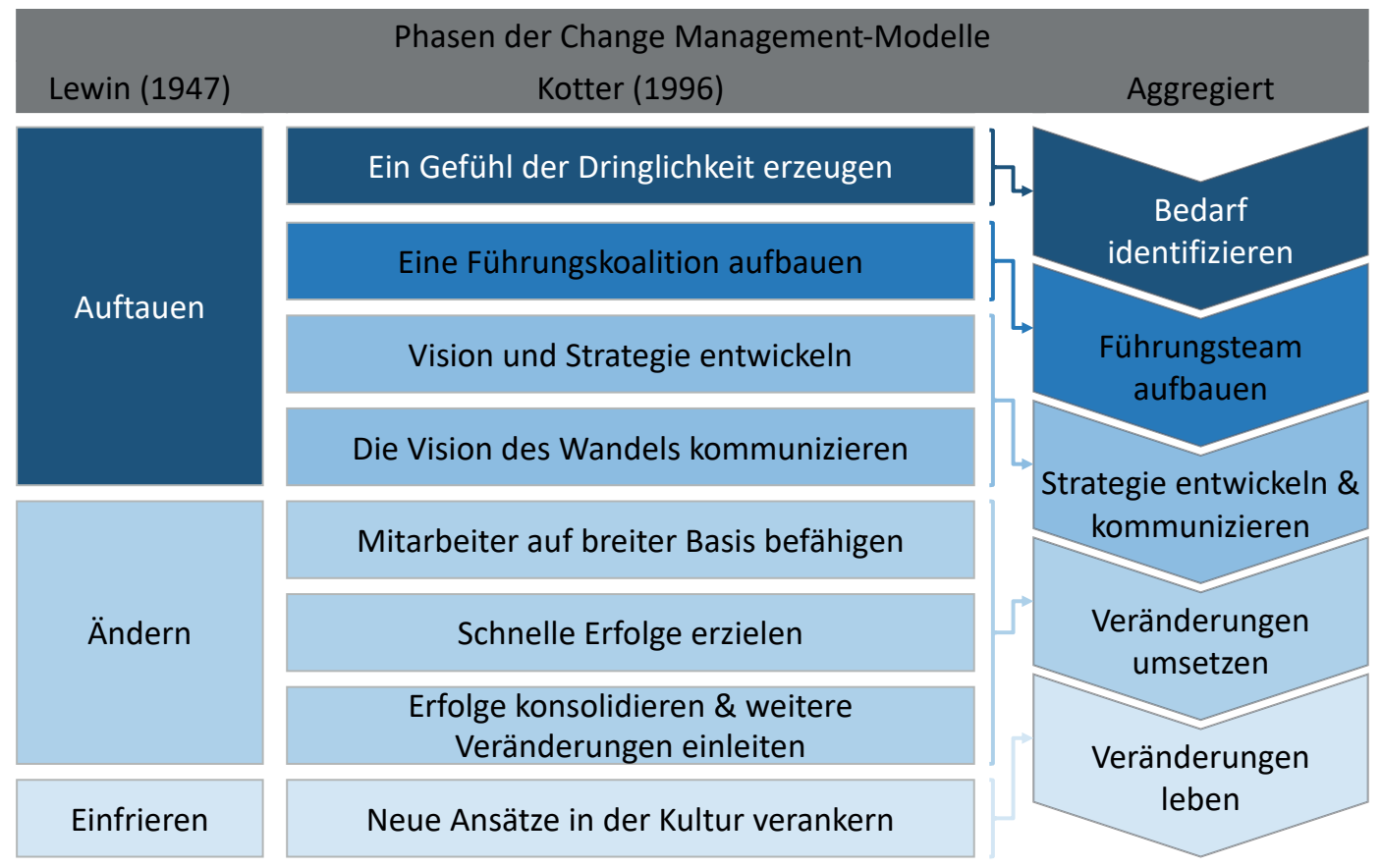

Bild 2: Phasen des Change Managements (eigene Darstellung).

Literatur

[1] Gentemann, L.: Deutsche Wirtschaft läuft der Digitalisierung weiter hinterher. URL: www.bitkom-research.de/ de/pressemitteilung/deutsche-wirtschaft-laeuft-der-digitalisierung-weiter-hinterher-0, Abrufdatum 16.09.2020

[2] Kersten, W:i von See, B.; Lodemann S.; Grotemeier, $C$ Trends und Strategien in LOgistik und Supply Chain Management - Entwicklungen und Perspektiven einer nachhaltigen und digitalen Transformation. BVL.digital GmbH: Bremen 2020.

[3] Stöcker C. Büch M. Sandner, P.: Die positive Seite dieser Krise. In: Frankfurter Allgemeine (2020). URL: https://www.faz.net/aktuell/ wirtschaft/digitec/chancen-in-der-pandemie-die-positive-seite-die-corona-krise-16733515.html, Abrufdatum: 20.08.2020.

[4] Plattform Industrie 4.0, $\mathrm{Ar}$ beitsgruppe 6 "Digitale Geschäftsmodelle": "Zehn Thesen zur Zukunft digitaler Geschäftsmodelle für Industrie 4.0 in der Post-Corona-Ökonomie. URL: www. plattform-i40.de/PI40/Redaktion/DE/Downloads/Publikation/Corona_Thesen.pdf? blob=publicationfile\&v $=\overline{5}$ Abrufdatum 16.09.2020.

[5] Kostka, C.: Change Management - Das Praxisbuch für Führungskräfte. München 2016. macher u. a. [17] neun relevante Dimensionen für eine erfolgreiche digitale Transformation: Strategie, Kultur, Führung, Mitarbeiter, Kunden, Governance, Produkte, Technologie und Prozesse. Ohne im Folgenden explizit auf jede dieser Dimensionen einzugehen, lässt sich bereits erkennen, dass die vier Erstgenannten die größten Überschneidungen zwischen den Themenfeldern Change Management und digitale Transformation aufweisen (bei der hier angeführten prozessualen Betrachtung des Change Managements). Bei der Betrachtung von Ausführungen verschiedener Autoren zu Teilbereichen dieser Dimensionen lässt sich feststellen, dass die fünf hier aufgezeigten Change Management-Phasen nicht alle notwendigen Aspekte ausreichend adressieren [7-9]. Das klassische Change Management ist im Zeitalter der digitalen Transformation daher nicht ausreichend und bedarf einer entsprechenden Anpassung.

\section{Erfolg durch Führung, Kommunikati- on und Mitarbeitereinbindung}

Um das Change Management adäquat anpassen zu können, bedarf es u. a. der Berücksichtigung bestehender Herausforderungen sowie Erfolgsfaktoren. Bild 3 liefert einen Überblick über die in der Befragung jeweils meistgewählten Punkte.

Wie dem Bild entnommen werden kann, sehen die meisten Befragten die Koordination der
Zusammenarbeit (58,7\%), gefolgt von zeitlichen Restriktionen (42,8 \%) und der Informations- und Berichterstattung (40,8\%) als die drei größten Herausforderungen des Change Managements in der digitalen Transformation. Mehr Einigkeit herrscht unterdessen bei den Erfolgsfaktoren. So sehen mehr als $85 \%$ aller Befragten die richtige Kommunikation als größten Erfolgsfaktor zur Umsetzung von Projekten an. An zweiter Stelle folgt die Partizipation der Mitarbeiter (76,5\%) und an dritter die richtige Führung im Wandel (74,2 \%).

In einer Detailbetrachtung dieser einzelnen $\mathrm{He}$ rausforderungen und Erfolgsfaktoren werden zum einen Überschneidungen untereinander deutlich (bspw. Koordination der Zusammenarbeit vs. richtige Führung etc.), zum anderen lassen sich die Faktoren den oben benannten Kernelementen - Strategie, Kommunikation und Mitarbeitereinbindung - zuordnen. Die empirische Untersuchung bestätigt demnach die auf der Literatur basierenden Kernelemente eines erfolgreichen Change Managements im Kontext der digitalen Transformation. Weiter lässt die Befragung die wesentliche Erkenntnis zu, dass die richtige Kommunikation auf sowie zwischen allen Ebenen einer der wichtigsten Aspekte für ein erfolgreiches Change Management ist, da diese mehr oder weniger Teil aller drei meistgewählten Erfolgsfaktoren ist. Darüber hinaus ist festzuhalten, dass die hier benannten Aspekte, ebenfalls unter Erfolgsfaktoren speziell für die digitale Transformation zu 
finden sind. Beispielsweise führt Harwardt im Rahmen des Erfolgsfaktors „Organisation“ an, dass eine verbesserte und schnellere Kommunikation für den Wandel zwingend notwendig ist und eine Studie von Fujistu aus 2018 mit über 1500 Teilnehmenden benennt Führung und Mitarbeiter als zwei der sechs zentralen Erfolgsfaktoren [18, 19].

Zuletzt ist anzumerken, dass für ein erfolgreiches Change Management in der digitalen Transformation mehr als nur die in der Befragung am höchsten priorisierten Erfolgsfaktoren ausschlaggebend sind. Vielmehr erscheint es sinnvoll, das Vorgehen der tradierten Modelle entsprechend der heutigen Bedarfe anzupassen. Ausgehend von dem oben dargestellten Prozess ist abzugleichen, welche Schritte bereits erfolgsversprechend sind und welche einer Erweiterung bedürfen. Auf Grundlage der Literatur und der Befragungsergebnisse sollte die aktive Mitarbeitereinbindung (a) ein zentraler Aspekt auf oberster Ebene des Modells sein (um diesem Punkt mehr Gewichtung zu verleihen) und weiter muss dieses selbst flexibler (b) werden. Zum besseren Verständnis: Auch in älteren Change-Modellen werden die Mitarbeiter ab einem gewissen Punkt eingebunden, allerdings häufig relativ spät und eher in einer passiven Rolle (sie werden informiert und haben wenig Gestaltungsmöglichkeiten). Bezüglich der Verbesserung der Flexibilität lässt sich u. a. die Kritik äußern, dass viele Modelle von einem langfristigen Endzustand ausgehen. Das mag bei Veränderungsprojekten in der Vergangenheit üblich gewesen sein. Bei aktuellen Digitalisierungsprojekten ist jedoch eher davon auszugehen, dass der erreichte Status quo nach erfolgreicher Umsetzung eines
Change-Projekts unter Umständen nicht langfristig besteht bzw. bestehen kann und demnach ein weiterführendes Projekt ggf. zeitnah starten muss. Zumindest ausgehend von der Annahme, dass die Digitalisierung eine höhere Anzahl an Veränderungsprojekten in kurzer Zeit bedingt [20].

\section{Das neugedachte Change Manage- ment im Unternehmen leben}

Um den aufgezeigten Anforderungen gerecht zu werden, können die oben benannten fünf Phasen der tradierten Change Management-Modelle erweitert bzw. angepasst werden. Zu (a): Unter der Einbindung der Mitarbeiter wird in diesem Zusammenhang "eine ernsthafte und nachhaltige Einbeziehung" verstanden, um einen größtmöglichen Erfolg erzielen zu können [9]. Das Ziel dieser Philosophie ist die frühestmögliche Einbeziehung aller Beteiligten, um diesen die Möglichkeit einer Einflussnahme zu geben, wodurch in der Folge eine gesteigerte Identifikation mit dem Projekt bzw. der Organisation erreicht werden kann. Zu (b): Das Thema Flexibilität bezieht sich auf die Wandlungsfähigkeit (bzw. -bereitschaft) der Unternehmen. Es gilt einen Change Management-Ansatz zu schaffen, welcher im Anschluss an ein erfolgreiches Change-Projekt die Flexibilität bietet auch nach kurzer Zeit (Bedarf vorausgesetzt) wieder mit einem neuen Wandlungsprozess zu starten.

Auf Grundlage dieser Erkenntnisse wurden die oben dargestellten aggregierten fünf Phasen der tradierten Change Management-Modelle weiterentwickelt (Bild 4). Die fünf Ur-Phasen bleiben weitestgehend bestehen. Der Prozess

Bild 3: Top 3 Herausforderungen und Erfolgsfaktoren des Change Managements in der digitalen Transformation $(n=207)$.

\begin{tabular}{|c|c|c|}
\hline \multicolumn{3}{|c|}{... des Change Managements in der digitalen Transformation (in \%) } \\
\hline & $\begin{array}{l}\text { 1. Koordination der } \\
\text { Zusammenarbeit }(58,7)\end{array}$ & $\begin{array}{l}\text { 1. Richtige Kommunikation zur } \\
\text { Vermeidung / Überbrückung von } \\
\text { Widerständen }(85,3)\end{array}$ \\
\hline 00:01 & 2. Zeitliche Restriktionen $(42,8)$ & $\begin{array}{l}\text { 2. Betroffene Abteilungen / } \\
\text { Personen mitgestalten lassen }(76,5)\end{array}$ \\
\hline $\bar{z}$ & $\begin{array}{l}\text { 3. Information und } \\
\text { Berichterstattung }(40,8)\end{array}$ & $\begin{array}{l}\text { 3. Richtige Führung während des } \\
\text { Wandels }(74,2)\end{array}$ \\
\hline
\end{tabular}

[6] Kersten, W.; Schröder, $M$ : Indorf, M:: Potenziale der Digitalisierung für das Supply Chain Risikomanagement: Eine empirische Analyse. In: Seiter, $M$ : Grünert, L: Berlin, $S$. (Hrsg): Betriebswirtschaftliche Aspekte von Industrie 4.0. Wiesbaden 2017

[7] Bowersox, D. J.; Closs, D. Drayer, R. W.: The Digital Transformation: Technology and Beyond, in: Supply Chain Management Review 9 (2005) 1, S. 22-29.

[8] Kane G. C. Palmer, D: Philips, A. N.; Kiron, D.; Buckley, N. Strategy, not Technology, Drives Digital Transformation. MIT Sloan Management Review and Deloitte University Press. 2015. URL: wwW2. deloitte.com/content/dam/ Deloitte/fr/Documents/strategy/dup strategy-not-technology-drives-digital-transformation.pdf, Abrufdatum 18.04.2020.

[9] Lauer, T.: Change Management Berlin Heidelberg 2019

[10] Isern, J.; Pung, C.: Organizing for successful Change Management: A McKinsey global survey, The McKinsey Quarterly, June (2006), S. 1-8.

[11] Miller, D.: Successful change leaders: what makes them? What do they do that is diferent?. In: Journal of Change Management 2 (2002) 4, S. 359-68. 
[12] Beer, M.; Nohria, N.: Cracking the Code of Change. In: Harvard Business Review 78(3) (2000), S. 133-141.

[13] Kotter, J. P.: Accelerate!: How the most Innovative Companies Capitalize on Today's Rapid-fire Strategic - and Still Make Their Numbers. In: Harvard Business Review 90 (2012) 11, S. 44-58.

[14] Lewin, K.: Frontiers in Group Dynamics, in: Human Relations 1 (1947) 1, S. 5-41

[15] Kotter, J. P.: Leading Change. 5 Auflage. München 2018.

[16] Krüger, W.: Excellence in Change. Wiesbaden 2008

[17] Schumacher, A.; Erol, S.; Sihn W.: A Maturity Model for Assessing Industry 4.0 Readines and Maturity of Manufacturing Enterprises. In: Procedia CIRP 52 (2016), S. 161-166.

[18] Harwardt, M.: Management der digitalen Transformation - Eine praxisorientierte Einführung. Wiesbaden 2019.

[19] Fujitsu Future Insights: Global Digital Transformation Survey Report 2018. URL: https://www.fujitsu.com/downloads/GLOBAL/vision/2018/ download-center/FTSV2018 Survey_EN-1.pdf, Abrufdatum 05.12 .2020

[20] Bundesministerium für Wirtschaft und Energie: Industrie 4.0 und Digitale Wirtschaft - Impulse für Wachstum, Beschäftigung und Innovation. Berlin 2015.

[21] Koch, S.: Einführung in das Management von Geschäftsprozessen: Six Sigma, Kaizen und TQM. 2. Auflage. Berlin 2015.

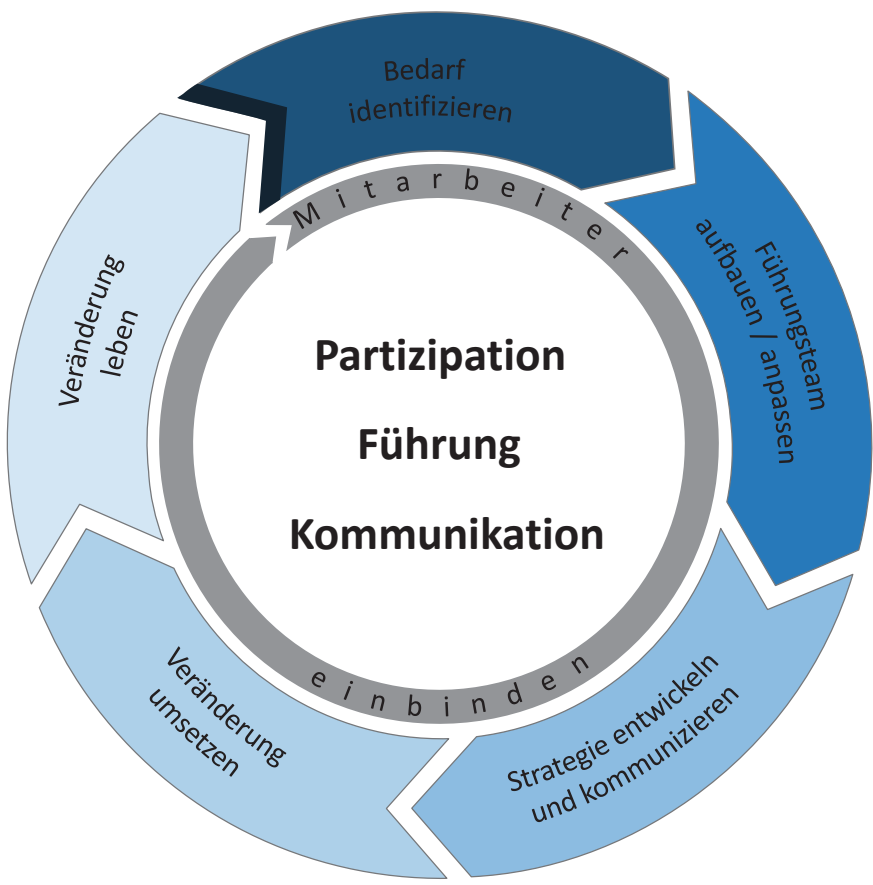

Bild 4: Transformation des Change Managements für die Digitalisierung.

hingegen wurde, in Anlehnung an den PDCA-Zyklus (ein Regelkreis aus dem Kontinuierlichen Verbesserungsprozess) in einen Regelkreis umgewandelt [21]. Die Aufgabe, im Anschluss an ein einzelnes Change-Projekt im Rahmen der digitalen Transformation weiterhin Bedarfe für den nächsten möglichen Wandel zu erkennen, wird somit betont. Das Zentrum des Regelkreises bilden die Mitarbeitereinbindung und die drei benannten Erfolgsfaktoren, welche nicht als optionale Elemente anzusehen sind. Den Erkenntnissen folgend, ist es - abgesehen von einzelnen, sehr spezifischen Führungsaufgaben (bspw. ein Führungsteam aufzubauen) - förderlich für den Erfolg von Change-Projekten, Mitarbeiter frühzeitig einzubinden (bspw. bereits bei der Identifikation von Bedarfen). In diesem Modell wurde daher auf eine explizite Verortung der Mitarbeitereinbindung zwischen zwei der fünf Ur-Phasen verzichtet, denn diese kann in allen Phasen erfolgsfördernd sein. Weiter sollen die Erfolgsfaktoren - Kommunikation, Partizipation und Führung - als wichtige rahmenschaffende Bedingungen in jedes Change-Projekt einfließen, um einen größtmöglichen Erfolg zu gewährleisten.

\section{Fazit und Ausblick}

Es lässt sich festhalten, dass das klassische Change Management in Unternehmen trotz seines relativ langen Bestehens und ohne den Fokus auf die digitale Transformation zu legen, bereits Optimierungsbedarfe aufweist. Ausge- hend von der anzunehmenden höheren Anzahl an Veränderungen (bedingt durch die digitale Transformation) wird deutlich, dass es nicht mehr lediglich eine Option darstellt, das Change Management in Unternehmen auszubauen. Es muss vielmehr eine Grundvoraussetzung für Führungskräfte werden, diesem Thema noch mehr Gewichtung zuzuteilen. Die hier primär diskutierten wichtigen Punkte eines Change Management-Ansatzes lassen sich teilweise auch in den tradierten Modellen erkennen, finden dort jedoch weniger Gewichtung. Darüber hinaus mag die Umsetzung einzelner Teile aus dem hier vorgestellten Modell - bspw. die Mitarbeitereinbindung - zunächst relativ einfach erscheinen, allerdings bedeutet dies in wahrscheinlich vielen Unternehmen einen grundlegenden Kulturwandel. Eine derartige Veränderung ist nicht kurzfristig umsetzbar - die Führungsebenen müssen vorab passende Kompetenzen erlernen und entsprechende Erfahrungen sammeln, um den Wandel bestmöglich unterstützen zu können.

Weiter ist der Hinweis zu geben, dass das hier vorgestellte angepasste Change Management-Modell lediglich eine grobe Richtung mit wichtigen, zu beachtenden Punkten vorgibt. Es bleibt Aufgabe der Unternehmen, die Schritte individuell anzupassen und im Zweifelsfall zu erweitern, spätestens wenn Themen wie bspw. Mitarbeitereinbindung aktuell nicht berücksichtig werden - sofern es bisher überhaupt eine Art Standard-Vorgehen für Veränderungsprojekte gibt.

Der hier vorgestellte Change Management-Ansatz wird im Rahmen des Projekts "Mittelstand 4.0-Kompetenzzentrum Hamburg" (Förderkennzeichen 01MF16003B; Projektträger Deutsches Zentrum für Luft und Raumfahrt) genutzt. Das Projekt ist Teil der Förderinitiative "Mittelstand 4.0 - Digitale Produktions- und Arbeitsprozesse" und wird im Rahmen des Förderschwerpunkts "Mittelstand-Digital - Strategien zur digitalen Transformation der Unternehmensprozesse" vom Bundesministerium für Wirtschaft und Energie aufgrund eines Beschlusses des deutschen Bundestages gefördert.

Schlüsselwörter:

Veränderungsmanagement, Digitalisierung, Umsetzung 